\title{
Intracranial Error Detection via Deep Learning
}

\author{
Martin Völker \\ Graduate School of Robotics \\ Albert-Ludwigs-University \\ Freiburg, Germany \\ martin.voelker@uniklinik-freiburg.de
}

\author{
Jiří Hammer \\ Department of Neurology \\ Motol University Hospital, Charles University \\ Prague, Czech Republic \\ jiri.hammer@lfmotol.cuni.cz
}

Joos Behncke

Department of Computer Science

Albert-Ludwigs-University

Freiburg, Germany

joos.behncke@uniklinik-freiburg.de

\author{
Lukas D.J. Fiederer \\ Faculty of Biology \\ Albert-Ludwigs-University \\ Freiburg, Germany \\ lukas.fiederer@uniklinik-freiburg.de
}

\author{
Robin T. Schirrmeister \\ Translational Neurotechnology Lab \\ University Medical Center Freiburg \\ Freiburg, Germany
}

robin.schirrmeister@uniklinik-freiburg.de

Andreas Schulze-Bonhage

Epilepsy Center

University Medical Center Freiburg

Freiburg, Germany

andreas.schulze-bonhage@uniklinik-freiburg.de

\author{
Petr Marusič \\ Department of Neurology \\ Motol University Hospital, Charles University \\ Prague, Czech Republic \\ petr.marusic@lfmotol.cuni.cz
}

\author{
Wolfram Burgard \\ Department of Computer Science \\ Albert-Ludwigs-University \\ Freiburg, Germany \\ burgard@informatik.uni-freiburg.de
}

\author{
Tonio Ball \\ Translational Neurotechnology Lab \\ University Medical Center Freiburg \\ Freiburg, Germany \\ tonio.ball@uniklinik-freiburg.de
}

\begin{abstract}
Deep learning techniques have revolutionized the field of machine learning and were recently successfully applied to various classification problems in noninvasive electroencephalography (EEG). However, these methods were so far only rarely evaluated for use in intracranial EEG. We employed convolutional neural networks (CNNs) to classify and characterize the error-related brain response as measured in 24 intracranial EEG recordings. Decoding accuracies of CNNs were significantly higher than those of a regularized linear discriminant analysis. Using time-resolved deep decoding, it was possible to classify errors in various regions in the human brain, and further to decode errors over $200 \mathrm{~ms}$ before the actual erroneous button press, e.g., in the precentral gyrus. Moreover, deeper networks performed better than shallower networks in distinguishing correct from error trials in all-channel decoding. In single recordings, up to $100 \%$ decoding accuracy was achieved. Visualization of the networks' learned features indicated that multivariate decoding on an ensemble of channels yields related, albeit non-redundant information compared to single-channel decoding. In summary, here we show the usefulness of deep learning for both intracranial error decoding and mapping of the spatio-temporal structure of the human error processing network.
\end{abstract}

Index Terms-Error Processing, Intracranial EEG, Deep Learning, BCI, Convolutional Neural Networks, Error Prediction

\section{INTRODUCTION}

Neurotechnological applications such as brain-computer interfaces (BCIs) can be improved by error decoding in electroencephalography (EEG) [1]-[5]. In addition to noninvasive recording techniques, intracranial EEG was also shown to be useable for error decoding $[6]-[\overline{8}]$.

This work was supported by DFG grant EXC1086 BrainLinks-BrainTools, Baden-Württemberg Stiftung grant BMI-Bot, Graduate School of Robotics in Freiburg, Germany and the State Graduate Funding Program of BadenWürttemberg, Germany.
However, when it comes to real-life applications, a high accuracy is decisive for the usefulness of such error decoding. In recent years, deep learning has driven the state-of-the-art of decoding accuracies in various fields of research [9], [10], especially in computer vision [11] and speech recognition [12] or generation [13]. Newly, deep learning techniques have also been successfully applied to an increasing number of decoding problems in EEG [14]-[18] and further utilized for extraction and visualization of learned features [19]. We previously reported that convolutional neural networks (CNNs) performed better than regularized linear discriminant analysis (rLDA) and filter bank common spatial patterns (FBCSP) algorithms in error decoding from noninvasive EEG [20], and can be used to reliably classify errors in inter-subject decoding [21]. From different CNN architectures, residual neural networks (ResNets) are a particularly promising architecture for challenging classification problems. ResNets employ a specialized CNN architecture with a typically very large number of convolutional layers [22], and have only recently been applied for classification in noninvasive EEG [14], [23]. Deep neural networks have also been successfully applied in the classification or prediction of epileptic signals [24]-[28] and movements [29], [30]. Other than that, deep learning techniques for BCIs based on intracranial recordings are so far mostly unexplored. Here we show for the first time the usefulness of CNNs and ResNets for both intracranial error decoding and as a tool for precise spatio-temporal brain mapping.

\section{EXPERIMENT}

In Freiburg, Germany, and in Prague, Czech Republic, 23 patients who were implanted with intracranial electrodes due 
to pharmacoresistant epilepsy participated in a flanker task experiment as described in [31]. One patient participated in two recording sessions on different days with a different subset of the intracranial electrodes; thus, 24 recording sessions were available overall. All patients gave their written informed consent before participating in the study. The study was approved by the local Ethics Committees. Trials were epoched on the onset of the correct or erroneous movement with the left or right index finger, as measured by analog joystick buttons. On average, patients executed $212 \pm 12$ (mean \pm SEM) correct and $51 \pm 5$ error trials and had an error rate of $19.34 \pm 1.79 \%$.

\section{Preprocessing, Decoding \& Statistics}

Locations of the stereotactic depth electrodes were identified with the help of post-implantation MRI or CT as in [32] and transformed into the MNI coordinate space [33]. Each electrode was then assigned to a specific brain region by calculating cytoarchitectonic probabilistic maps in the SPM anatomy toolbox [34]. Intracranial EEG data were re-referenced bipolarly between the respective neighbors to be specific for local effects and reduce external noise contamination, and resampled to $250 \mathrm{~Hz}$. Other than that, the EEG data were only minimally pre-processed, as described in [21], to operate under application-oriented conditions. We used open-source python implementations for both rLDA [35] and CNN classifiers. Deep4Net and ShallowNet architectures were employed as described in [14] and available in the Braindecode Toolbox ${ }^{1}$ Additionally, we used a 34-layered ResNet architecture ${ }^{2}$ and the compact EEGNet architecture was reimplemented as described in [36], as the EEGNet code from the original publication was not available. As optimizer, we used AdamW [37] with cosine annealing [38], a weight decay of 0.002 and an initial learning rate of $\frac{0.01}{32}$. For each recording, the first $60 \%$ of the data was used for training, and the last $40 \%$ were reserved as final evaluation set, which was only used to test the final accuracies.

Statistical significance of the single-channel classifications was evaluated by randomly permuting the true labels of the test set $10^{6}$ times to generate a null distribution. For significance of brain region accuracy averages and classifier comparisons, a Wilcoxon signed rank test was employed [39].

The classification of errors is typically a problem with a strong trial imbalance, as correct trials occur far more often in realistic applications. Error decoding studies also usually report a higher classification accuracy for the correct class [40]. In a decoding problem with such a strongly imbalanced number of trials per class, it is thus advisable to define a normalized accuracy acc $_{\text {norm }}$ as:

$$
\text { acc }_{\text {norm }}=\frac{1}{n \text { Classes }} * \sum_{i=1}^{n \text { Classes }} \operatorname{TPR}\left(\text { class }_{\mathrm{i}}\right)
$$

with the True Positive Rate (TPR or sensitivity [41]) as:

${ }^{1}$ https://robintibor.github.io/braindecode/source/braindecode.models.html

2 https://github.com/robintibor/adamw-eeg-eval/blob/

445cc5d471d8eea3814ffa39621974dda7c471a6/adamweegeval/resnet.py

$$
\operatorname{TPR}\left(\text { class }_{\mathrm{i}}\right)=\frac{T P\left(\text { class }_{\mathrm{i}}\right)}{T P\left(\operatorname{class}_{\mathrm{i}}\right)+F N\left(\text { class }_{\mathrm{i}}\right)}
$$

$\mathrm{TP}$ is the number of true positives, and FN the number of false negative decoding results per class. Thus, when

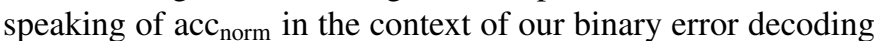
problem, we define it as arithmetic mean of TPR(correct) and TPR(error); this method is also known as macro-averaging [42]. Macro-averaging prohibits classifiers from achieving seemingly high accuracies by exploiting the trial imbalance, e.g., by only predicting the most abundant class for all trials, as

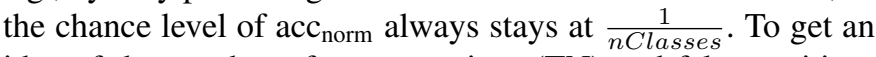
idea of the number of true negatives (TN) and false positives (FP), we also included the specificity (or true negative rate),

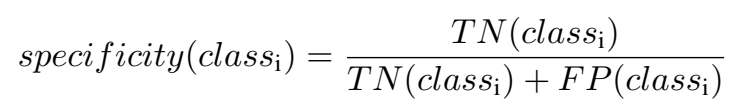

as well as the F1 score, i.e., the harmonic mean of precision and sensitivity, as further measures.

$$
F 1\left(\operatorname{class}_{\mathrm{i}}\right)=\frac{2 * T P\left(\operatorname{class}_{\mathrm{i}}\right)}{2 * T P\left(\operatorname{class}_{\mathrm{i}}\right)+F P\left(\operatorname{class}_{\mathrm{i}}\right)+F N\left(\text { class }_{\mathrm{i}}\right)}
$$

To cope with the class imbalance, we further used Braindecode's ClassBalancedBatchSizeIterator, which draws the training samples such that, in expectation, the same number of examples is drawn per class. Other metrics, like training accuracies, were also macro-averaged.

\section{COMPARISON OF ClasSIFIERS}

\section{A. Single-Channel Decoding}

Channel-selection in intracranial EEG is not trivial, especially as the electrode locations are unique in every measurement and do not follow a certain spatial pattern as is usually the case in noninvasive EEG. Therefore, we first compared the classifiers methods in single-channel decoding. Table I gives a comparison of classifier performance in this setting, evaluated with 200 training epochs.

TABLE I

CLASSIFIER PERFORMANCE IN SINGLE-CHANNEL DECODING. FOR BOTH SINGLE-CLASS AND NORMALIZED ACCURACY, MEAN \pm SEM ARE LISTED.

\begin{tabular}{|c|c|c|c|}
\hline Classifier & acc $_{\text {norm }} \mathbf{( \% )}$ & acc $_{\text {correct }}(\%)$ & acc $_{\text {error }}$ (\%) \\
\hline Deep4Net & $59.28 \pm 0.50$ & $69.37 \pm 0.44$ & $49.19 \pm 0.56$ \\
ShallowNet & $58.42 \pm 0.32$ & $74.83 \pm 0.25$ & $42.01 \pm 0.40$ \\
EEGNet & $57.73 \pm 0.52$ & $57.78 \pm 0.48$ & $57.68 \pm 0.56$ \\
rLDA & $53.76 \pm 0.32$ & $76.12 \pm 0.26$ & $31.40 \pm 0.38$ \\
ResNet & $52.45 \pm 0.21$ & $95.47 \pm 0.14$ & $09.43 \pm 0.28$ \\
\hline
\end{tabular}

In single-channel decoding, the Deep4Net had the best normalized accuracy. This difference was significant with $\mathrm{p}<0.001$ (Wilcoxon signed-rank test) in regard to all other methods. For the Deep4Net, we thus visualized the single channel accuracies in Fig. 11 The broad distribution of electrodes with high accuracies indicates that errors were not only decodable from limited brain regions; rather, there seems to 


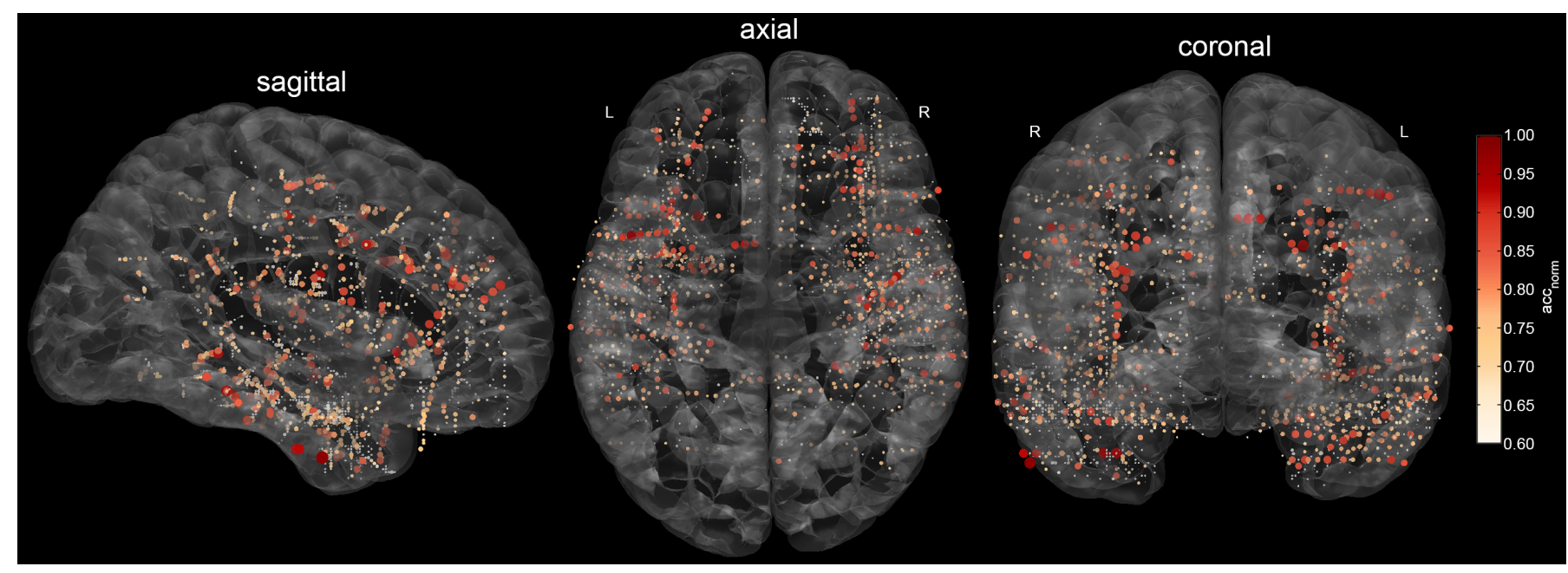

Fig. 1. Single-channel decoding using the Deep4Net on a $2 \mathrm{~s}$-window $(-0.5 \mathrm{~s}$ to $1.5 \mathrm{~s}$ in regard to the button press event) of intracranial EEG data. All 2332 channels are marked according their MNI coordinates in an ICBM152 brain template [43]; normalized accuracies $>60 \%$ are plotted color- and size-coded.
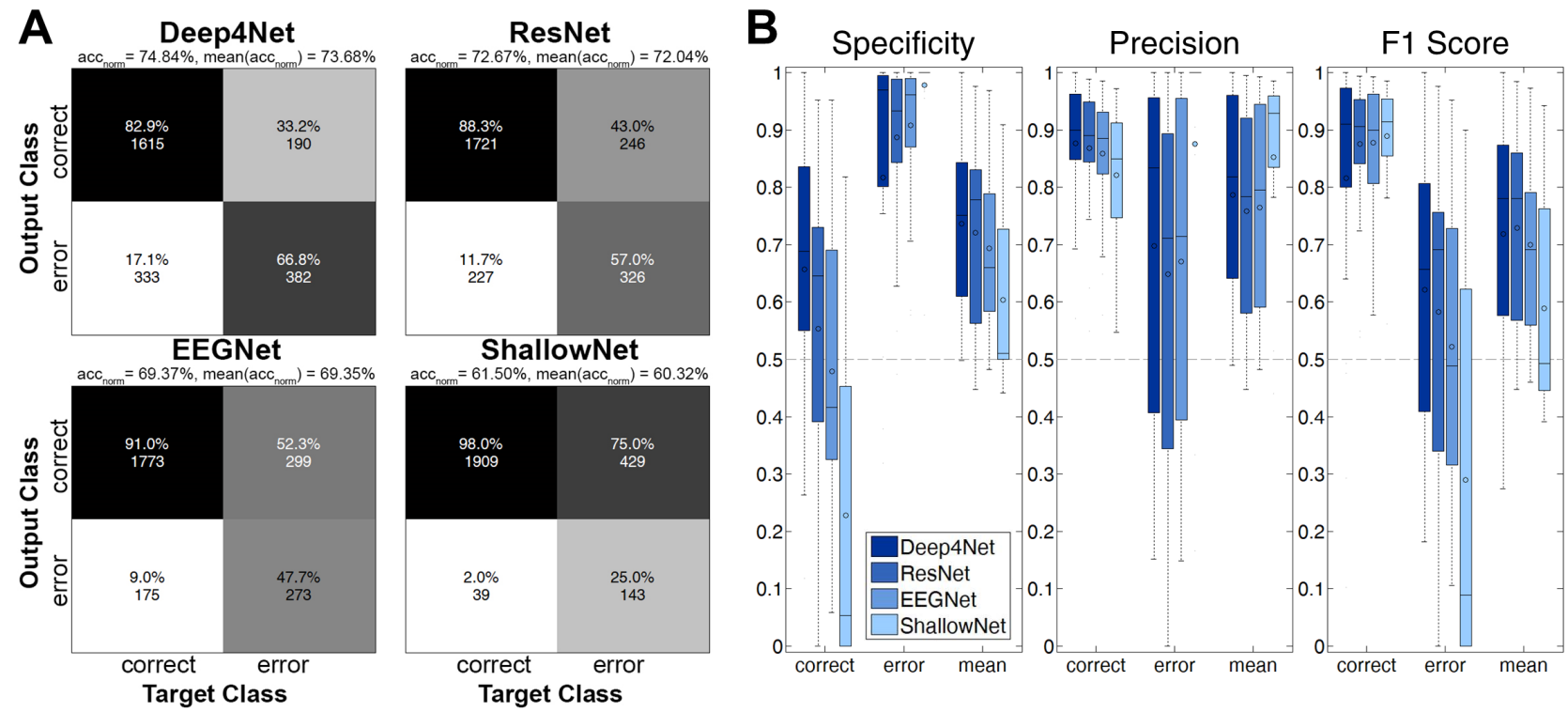

Fig. 2. Classifier performance in all-channel decoding. Here, the classifiers were trained on all available channels per patient. A) Confusion matrices of the four models used for decoding. The matrices display the sum of all trials over the 24 recordings. On top of the matrices, the normalized accuracy over all trials, i.e., acc $\mathrm{n}_{\text {norm}}$, and the mean of the single recordings' normalized accuracy, i.e., mean ( acc $_{\text {norm}}$ ) is displayed; please note that these two measures differ slightly, as the patients had a varying number of total trials and trials per class. B) Box plots for specificity, precision and F1 score. The box represents the interquartile range (IQR) of the data, the circle within the mean, the horizontal line depicts the median. The lower whiskers include all data points that have the minimal value of $25^{\text {th }}$ percentile- $1.5 * I Q R$, the upper whiskers include all points that are maximally $75^{\text {th }}$ percentile $+1.5 * I Q R$.

be an extended error-processing network, and the CNN was able to classify errors in multiple nodes within this network. All in all, electrodes in central and frontal regions had higher accuracies than electrodes in parietal locations.

\section{B. Decoding Using All Channels}

As individual channels may carry non-redundant information, multivariate decoding from all available channels simultaneously may increase decoding performance. We compared network architectures in this application scenario (Fig.2]A) using 1000 training epochs. Here, the Deep4Net performed best with an normalized accuracy of $74.84 \%$, ResNet $(72.67 \%)$, and EEGNet $(69.37 \%)$ followed, while the ShallowNet was far behind $(61.50 \%)$. Measures of specificity, precision, and the F1 score per class (Fig. 2 B), indicate that especially the two smaller networks (i.e., networks with fewer parameters, EEGNet and ShallowNet) tended to overpredict the "correct" class, leading to low sensitivity for the error class. The average F1 score for the ShallowNet was significantly $(\mathrm{p}<0.01$, Wilcoxon signed-rank test) lower than that for the other 3 architectures. We plotted the confusion matrices of the single recordings for the Deep4Net in Fig. 3 While there were 

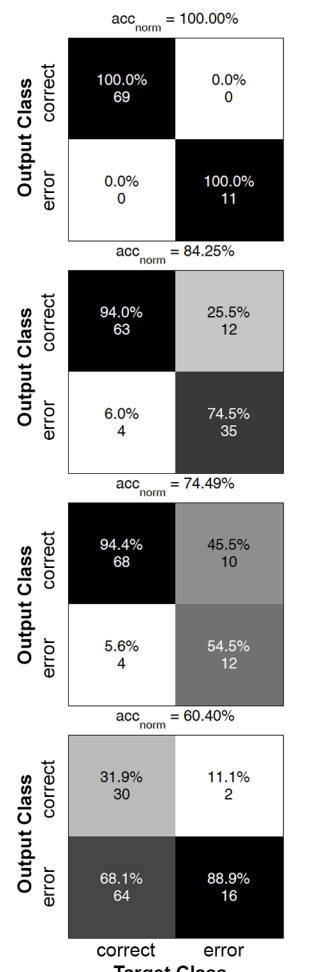
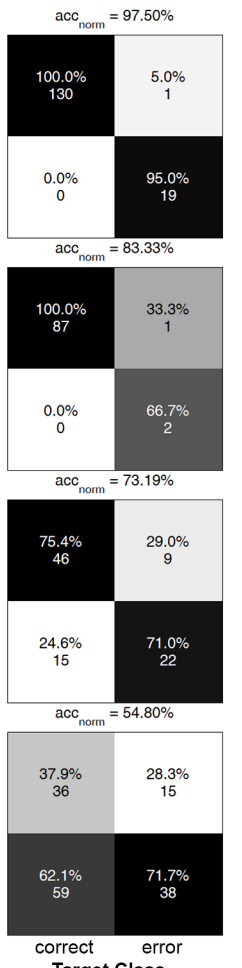
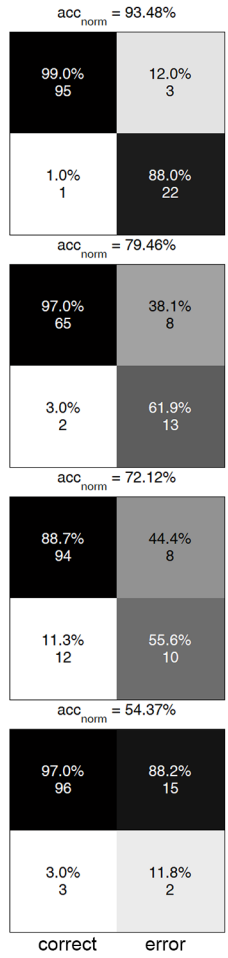
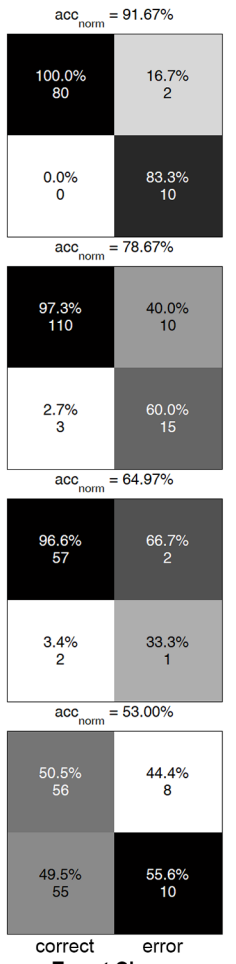
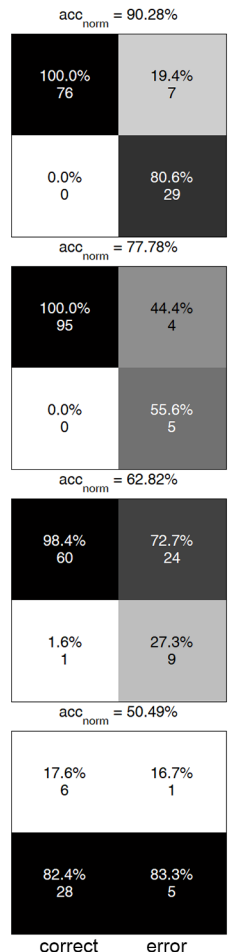

Target Class
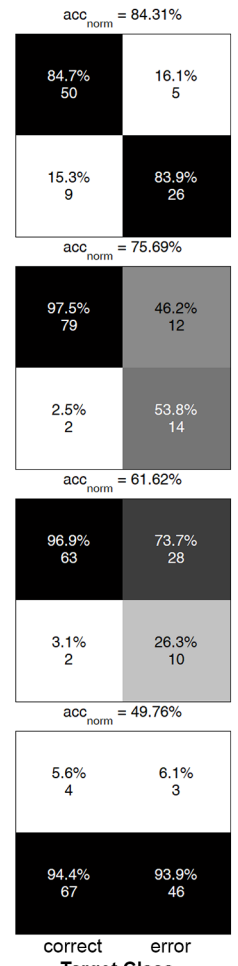

Target Class

Fig. 3. Single-recording confusion matrices of the Deep4 network performing all-channel error decoding. The matrices are sorted in a descending order according to the normalized decoding accuracy. On top of each confusion matrix, the corresponding normalized accuracy is specified.

recordings in which the CNN performed with $100 \%$ or very close to perfect accuracy, there were also some in which the classifier predicted nearly only one class and thus performed with an acc norm $_{\text {around }} 50 \%$. There could be multiple reasons for that. First, as the location of the electrodes is determined by the epilepsy focus, it could be that those recordings did not include any useful locations for error decoding. Second, it could be that a strong class imbalance led to an overfitting on one class. Third, some recordings had very little training data, and one could assume that the CNN was not able to learn the class representations reliably. However, using Spearman's rank correlation, the decoding performance did neither significantly correlate with the number of errors $(\mathrm{r}=0.23, \mathrm{p}=0.28)$, the correct trials in the training data $(\mathrm{r}=-0.07, \mathrm{p}=0.75)$, nor the total error rate $(\mathrm{r}=-0.05, \mathrm{p}=0.82)$.

Generally, it is a known problem in the field of error decoding that classifiers have a higher sensitivity on the correct class. It is believed that the strong trial imbalance could be one reason for this issue [40]. We used a class-balanced batch size iterator to reduce this problem; but as this method can repeatedly choose samples of the less frequent class, it could theoretically still overfit on this class. To test for that, we repeated the classification with the Deep4Net, while keeping the number of trials of each class in training and test set equal (Fig. 4). For each error trial, the nearest correct trial (randomly before or after) was chosen to keep. In that way, any changes over the time of the experiment could not influence the decoding; otherwise the CNN might mistakenly associate

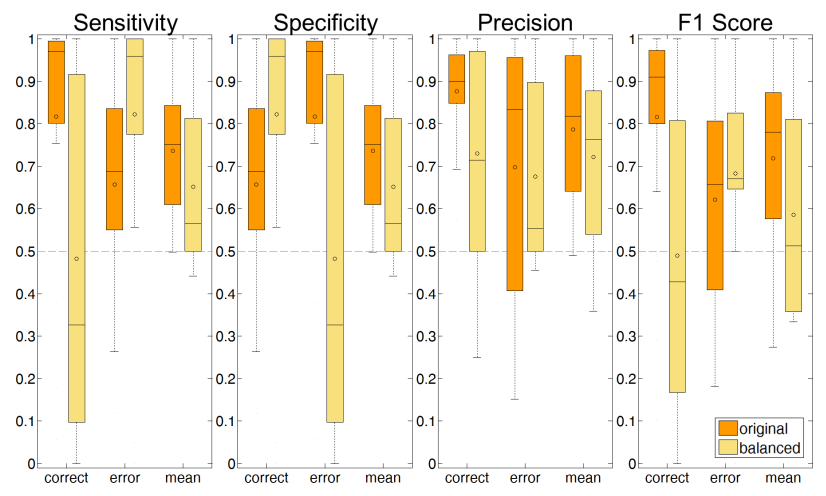

Fig. 4. All-channel decoding (Deep4Net) on the original, imbalanced data and a balanced dataset using sub-sampling of the more frequent class.

unrelated slow changes in the EEG signal with the class that was more prominent during that time of the recording.

Interestingly, after trial balancing, the sensitivity for the correct class decreased distinctly, while the sensitivity for the error class increased. However, the precision did not improve for the error class, while it decreased for the correct class. The F1 score of the correct class decreased strongly, while the error class saw only little improvement. The average F1 score decreased. All in all, it does not seem to be advisable to balance the number of trials by sub-sampling. 

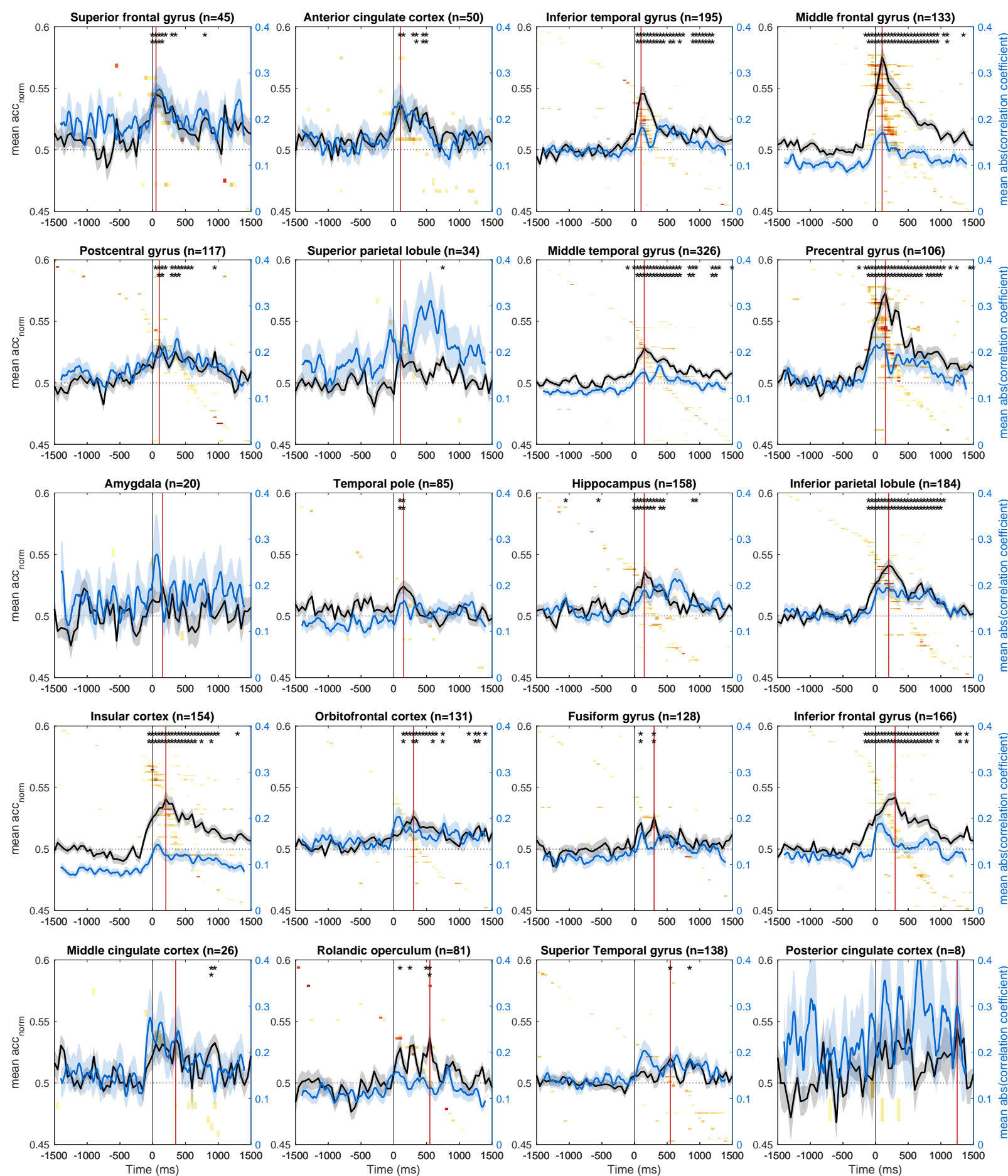

Fig. 5. Single-channel decoding using the Deep4Net in a time-resolved manner. Per area, significant $(\mathrm{p}<0.01$, permutation test) single-channel accuracies are plotted color-coded in the background. The black curve displays the mean normalized decoding accuracy in the respective area, as calculated within a $200 \mathrm{~ms}$ sliding window (50 ms step size), and the standard error of the mean (SEM) is plotted semitransparent. Time points with accuracies significantly higher than the the $50 \%$ chance level are highlighted by asterisks $(*=\mathrm{p}<0.001$, $* *=\mathrm{p}<0.0001$, Wilcoxon signed-rank test). The plots were sorted according to the time point of the highest mean accuracy per area (red line). The blue curve represents a visualization of the CNN's learned attributes, as calculated by time-resolved voltage feature input-perturbation network-prediction correlation mapping. Analog to the $200 \mathrm{~ms}$ decoding window, we applied a moving-average filter with a $200 \mathrm{~ms}$ gaussian window on the correlation values. 

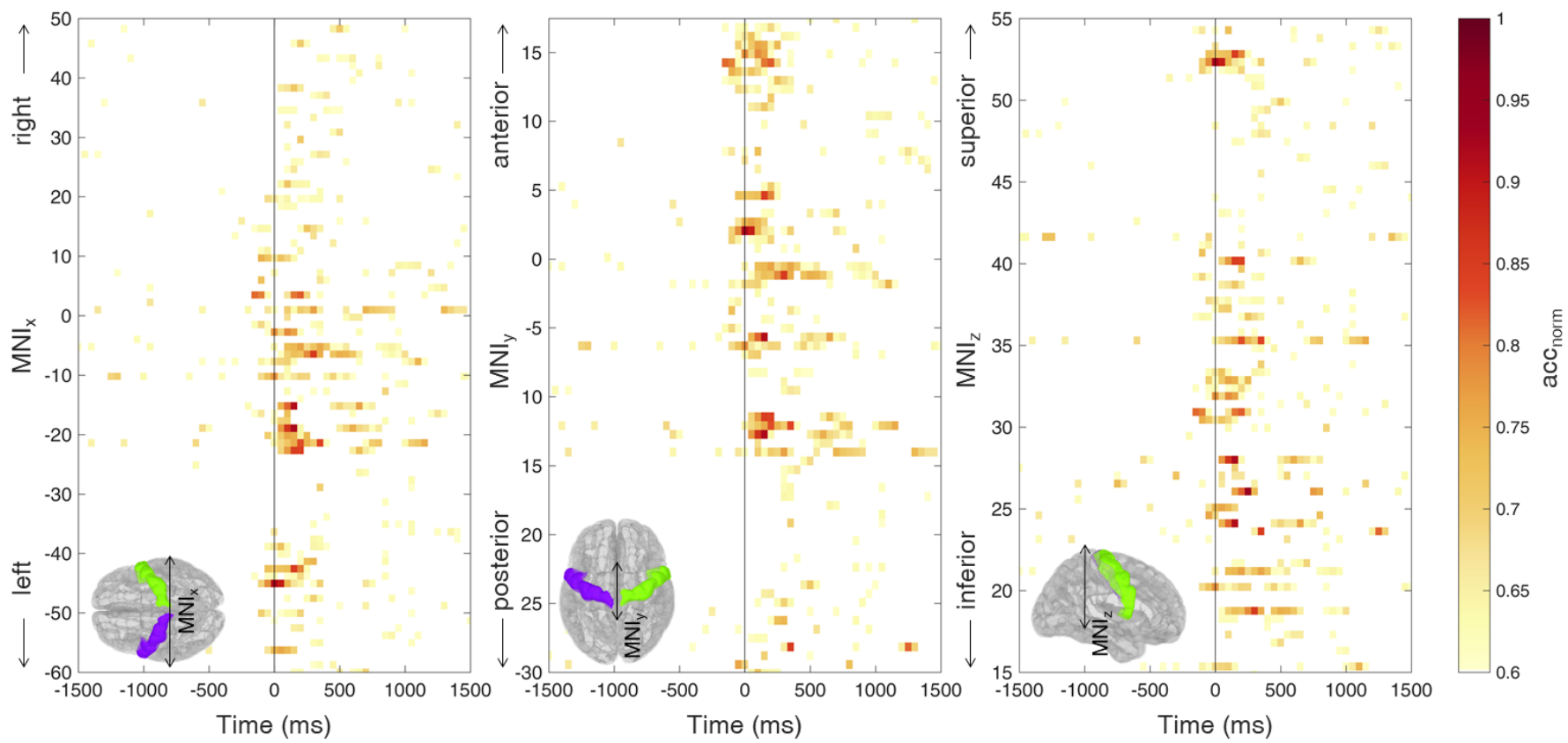

Fig. 6. Time-resolved normalized decoding accuracy in the precentral gyrus in relation to the spatial distribution of channels in 3D space. In the bottom left corners, the precentral gyrus was visualized with Brainstorm [44] with the respective orientation (left side purple, right side green).

\section{VISUALIZING THE SPATIO-TEMPORAL PROPERTIES OF THE ERROR RESPONSE}

To further dissect the temporal evolution of the errorrelated response, we used a shifting $200 \mathrm{~ms}$ window on each channel for decoding with the Deep4Net. Due to the short time window, we had to deviate here from the default network parameters and use a stride of 2 samples, as well as reduced filter time lengths of 2 samples. Moreover, time-resolved voltage feature input-perturbation network-prediction correlations were calculated to visualize the network's learned features (detailed description in [14], [19]).

Importantly, the decoding results were obtained via singlechannel decoding, while the input-perturbation networkprediction correlations were calculated on a model trained on all channels of the respective patient. To ensure a proper comparison of the two methods, we used the same network parameters as in the shifting-window classification for this analysis. Results from electrodes in each of 20 regions of interest (ROIs) were then pooled and illustrated in Fig. 5.

In the precentral gyrus, it was possible to significantly $(\mathrm{p}<0.001$ in the region mean) decode errors $250 \mathrm{~ms}$ (window center) before the actual button-press event; In the middle and inferior frontal gyrus, errors were significantly decodable $150 \mathrm{~ms}$ before the event. Activity in the hippocampus could even be predictive $1 \mathrm{~s}$ and $0.5 \mathrm{~s}$ before an error.

Generally, the peak of maximal decodability shifted from frontal to parietal and temporal brain regions over the time course of the error response. The reduced stride and filter time lengths resulted in lower total decoding accuracies; nevertheless, for comparison of the spatio-temporal distribution of the error response, this proved to be a useful method.
Input-perturbation network-prediction correlations (Fig. 5 blue curves) and time-resolved decoding accuracies shared a similar development over time, although there are some differences, e.g., in the time point of the peak and sharpess of the slope, as seen in the inferior frontal gyrus or in the superior temporal gyrus.

In contrast to the single-channel decoding accuracies, the highest normalized network-prediction correlation values were found at parietal sites, such as the superior parietal lobule. This could indicate that parietal sites rely more on large-scale network-based activity during error processing, which is not easily decodable from single channels.

Similar to the accuracies' development over time, activities at frontal and precentral areas, such as the precentral gyrus and the middle frontal gyrus, were the first to exhibit a sharp increase of the correlation values before the actual error event, indicating that the network learned these to be predictive to the event outcome.

As the precentral gyrus was the most promising area for the detection of errors prior to the erroneous event, we further sorted the single channel accuracies according to their spatial distribution in the MNI space, to examine which exact parts of the precentral gyrus are activated earliest (Fig. 6).

Distinctively, activity in the anterior parts of the precentral gyrus was predictive for errors earlier than in the posterior part. This fits the observation that frontal regions were also activated slightly before the error event. Moreover, central parts of the precentral gyrus were predictive earlier than lateral (left and right) regions. This hints toward a central origin of the error response, which is then distributed to lateral parts of the motor system. 


\section{DISCUSSION}

\section{A. Classifier Comparison}

When decoding on all channels, especially deeper networks strongly gained accuracy compared to single-channel decoding. Notably, the ShallowNet performed second-best on singlechannel decoding and last in all-channel decoding, while the ResNet, which is the deepest of the tested architectures with 34 convolutional layers, performed worst in single-channel decoding, and second-best in all-channel decoding on all channels. One could thus speculate that deeper networks need a broader spectrum of data to perform reliably, while very shallow networks cannot cope with the amount of noise in allchannel decoding. The 4-layered Deep4Net, however, seems to be a good compromise, as it performed best in both modalities.

\section{B. Error Detection \& Prediction}

We have shown that CNNs are able to detect errors on a single-trial basis in intracranial recordings from precentral and frontal brain regions even before the actual error event, i.e., the false button press, happened. Especially activity in the precentral gyrus was predictive for errors at early time points. Even earlier decodability in the hippocampus could hint toward a role of memory recall for the task performance.

The predictability of errors is an active topic in many areas of neuroimaging; e.g., error-prone patterns were identified in fMRI [45], preceding errors up to $30 \mathrm{~s}$ in the default mode network (DMN), especially in the medial prefrontal cortex (mPFC). There, preparatory action in a prefrontal-extrastriate network is assumed to happen. Moreover, the contingent negative variation $(\mathrm{CNV})$, an event-related slow wave related to preparatory attention [46] was shown to be reduced from $100 \mathrm{~ms}$ before an actual error [47] and is assumed to be partly generated in the pre-supplementary motor area (pre-SMA). It has also been shown that the DMN can be mapped using intracranial measurements in humans, and that precentral and midfrontal regions are an important part of the human DMN [48]. In noninvasive EEG, activity at frontocentral scalp sites proximal to the anterior cingulate cortex (ACC) were shown to be predictive for errors [49]. The $\mathrm{mPFC}$ has further been linked to cognitive control dynamics during action monitoring [50] in a flanker task. The motor cortex is further involved in predictive coding of future movements [51], and it has been shown that premotor and motor cortices encode expected rewards [52]. Moreover, activity in the mPFC, the prefrontal cortex and motor cortex might serve as a top-down control signal that inhibits inappropriate responding [53].

Thus, our results fit very well in the involvement of frontal and precentral brain regions in action control and preparatory motor planning.

\section{CONCLUSION}

Here we have shown that deep learning methods, especially deep convolutional neural networks including residual neural networks, are not only among the best available machine learning methods for various decoding problems, but are also an invaluable tool for brain mapping, as shown in human intracranial recordings during error processing. By using slidingwindow short-time decoding on single channels, we characterized the intracranial error response in depth; in comparison with the visualization of learned attributes in all-channel decoding, we could further show that both methods reveal overlapping, but not similar information, hinting towards the ability of CNNs to extract hidden connectivity features.

Deep convolutional networks were very accurate in allchannel decoding of errors from intracranial EEG electrodes in epilepsy patients, even though many of the channels were not informative. More compact or shallower networks, however, were prone to overfitting and not able to solve this challenging task reliably. For the intracranial classification of errors, we thus would recommend the use of at least 4-layered CNNs to reach an adequate number of parameters and thereby sufficient ability to learn.

\section{ACKNOWLEDGMENT}

The authors would like to thank Pavel Kršek, Martin Tomášek, Peter C. Reinacher and Volker A. Coenen for their support, and the involved patients for their participation.

\section{REFERENCES}

[1] M. Spüler, M. Bensch, S. Kleih, W. Rosenstiel, M. Bogdan, and A. Kübler, "Online use of error-related potentials in healthy users and people with severe motor impairment increases performance of a p300bci," Clinical Neurophysiology, vol. 123, no. 7, pp. 1328-1337, 2012.

[2] I. Iturrate, L. Montesano, and J. Minguez, "Shared-control braincomputer interface for a two dimensional reaching task using eeg errorrelated potentials," in 35th Annual International Conference of the IEEE Engineering in Medicine and Biology Society (EMBC). IEEE, 2013, pp. 5258-5262.

[3] R. Chavarriaga, I. Iturrate, Q. Wannebroucq, and J. d. R. Millán, "Decoding fast-paced error-related potentials in monitoring protocols," in 37th Annual International Conference of the IEEE Engineering in Medicine and Biology Society (EMBC). IEEE, 2015, pp. 1111-1114.

[4] A. Kreilinger, C. Neuper, and G. R. Müller-Putz, "Error potential detection during continuous movement of an artificial arm controlled by brain-computer interface," Medical \& biological engineering \& computing, vol. 50, no. 3, pp. 223-230, 2012.

[5] C. L. Dias, A. I. Sburlea, and G. R. Müller-Putz, "Masked and unmasked error-related potentials during continuous control and feedback,' Journal of Neural Engineering, 2018.

[6] T. Milekovic, T. Ball, A. Schulze-Bonhage, A. Aertsen, and C. Mehring, "Detection of error related neuronal responses recorded by electrocorticography in humans during continuous movements," PloS one, vol. 8, no. $2,2013$.

[7] J. Wander, J. Olson, J. Ojemann, and R. Rao, "Cortically-derived errorsignals during bci use," in Proceedings of the 5th International BrainComputer Interface Meeting, 2013.

[8] N. Even-Chen, S. D. Stavisky, J. C. Kao, S. I. Ryu, and K. V. Shenoy, "Augmenting intracortical brain-machine interface with neurally driven error detectors," Journal of neural engineering, vol. 14, no. 6, p. 066007 , 2017.

[9] Y. LeCun, Y. Bengio, and G. Hinton, "Deep learning," nature, vol. 521, no. 7553 , p. $436,2015$.

[10] I. Goodfellow, Y. Bengio, A. Courville, and Y. Bengio, Deep learning. MIT press Cambridge, 2016, vol. 1.

[11] A. Krizhevsky, I. Sutskever, and G. E. Hinton, "Imagenet classification with deep convolutional neural networks," in Advances in neural information processing systems, 2012, pp. 1097-1105.

[12] D. Amodei, S. Ananthanarayanan, R. Anubhai, J. Bai, E. Battenberg, C. Case, J. Casper, B. Catanzaro, Q. Cheng, G. Chen et al., "Deep speech 2: End-to-end speech recognition in english and mandarin," in International Conference on Machine Learning, 2016, pp. 173-182. 
[13] A. Van Den Oord, S. Dieleman, H. Zen, K. Simonyan, O. Vinyals, A. Graves, N. Kalchbrenner, A. Senior, and K. Kavukcuoglu, "Wavenet: A generative model for raw audio," arXiv preprint arXiv:1609.03499, 2016.

[14] R. T. Schirrmeister, J. T. Springenberg, L. D. J. Fiederer, M. Glasstetter, K. Eggensperger, M. Tangermann, F. Hutter, W. Burgard, and T. Ball, "Deep learning with convolutional neural networks for EEG decoding and visualization." Human brain mapping, vol. 38, no. 11, pp. 53915420, Nov. 2017.

[15] J. Behncke, R. T. Schirrmeister, W. Burgard, and T. Ball, "The signature of robot action success in EEG signals of a human observer: Decoding and visualization using deep convolutional neural networks," in 6th International Conference on Brain-Computer Interface (BCI). IEEE, 2018.

[16] R. Schirrmeister, L. Gemein, K. Eggensperger, F. Hutter, and T. Ball, "Deep learning with convolutional neural networks for decoding and visualization of eeg pathology," in Signal Processing in Medicine and Biology Symposium (SPMB), 2017 IEEE. IEEE, 2017, pp. 1-7.

[17] M. J. Putten, S. Olbrich, and M. Arns, "Predicting sex from brain rhythms with deep learning," Scientific reports, vol. 8, no. 1, p. 3069, 2018.

[18] A. Sors, S. Bonnet, S. Mirek, L. Vercueil, and J.-F. Payen, "A convolutional neural network for sleep stage scoring from raw single-channel eeg," Biomedical Signal Processing and Control, vol. 42, pp. 107-114, 2018.

[19] K. G. Hartmann, R. T. Schirrmeister, and T. Ball, "Hierarchical internal representation of spectral features in deep convolutional networks trained for eeg decoding," in 6th International Conference on Brain-Computer Interface (BCI). IEEE, 2018.

[20] M. Völker, S. Berberich, E. Andreev, L. D. Fiederer, W. Burgard, and T. Ball, "Between-subject transfer learning for classification of errorrelated signals in high-density eeg," in The First Biannual Neuroadaptive Technology Conference, vol. 81, no. 8.8, 2017, p. 47.

[21] M. Völker, R. T. Schirrmeister, L. D. Fiederer, W. Burgard, and T. Ball, "Deep transfer learning for error decoding from non-invasive EEG," in 6th International Conference on Brain-Computer Interface (BCI). IEEE, 2018.

[22] K. He, X. Zhang, S. Ren, and J. Sun, "Deep residual learning for image recognition," in Proceedings of the IEEE conference on computer vision and pattern recognition, 2016, pp. 770-778.

[23] F. Wang, S.-h. Zhong, J. Peng, J. Jiang, and Y. Liu, "Data augmentation for eeg-based emotion recognition with deep convolutional neural networks," in International Conference on Multimedia Modeling. Springer, 2018, pp. 82-93.

[24] D. Krug, C. E. Elger, and K. Lehnertz, "A cnn-based synchronization analysis for epileptic seizure prediction: Inter-and intraindividual generalization properties," in 11th International Workshop on Cellular Neural Networks and Their Applications. IEEE, 2008, pp. 92-95.

[25] A. Antoniades, L. Spyrou, C. C. Took, and S. Sanei, "Deep learning for epileptic intracranial eeg data," in IEEE 26th International Workshop on Machine Learning for Signal Processing (MLSP). IEEE, 2016, pp. $1-6$.

[26] D. Ahmedt-Aristizabal, C. Fookes, K. Nguyen, and S. Sridharan, "Deep classification of epileptic signals," arXiv preprint arXiv:1801.03610, 2018.

[27] A. Antoniades, L. Spyrou, D. Martin-Lopez, A. Valentin, G. Alarcon, S. Sanei, and C. C. Took, "Detection of interictal discharges with convolutional neural networks using discrete ordered multichannel intracranial eeg," IEEE Transactions on Neural Systems and Rehabilitation Engineering, vol. 25, no. 12, pp. 2285-2294, 2017.

[28] M.-P. Hosseini, D. Pompili, K. Elisevich, and H. Soltanian-Zadeh "Optimized deep learning for eeg big data and seizure prediction bci via internet of things," IEEE Transactions on Big Data, vol. 3, no. 4, pp. 392-404, 2017.

[29] Z. Xie, O. Schwartz, and A. Prasad, "Decoding of finger trajectory from ecog using deep learning," Journal of neural engineering, vol. 15, no. 3 , p. 036009, 2018.

[30] X. R. N. Wang, A. Farhadi, R. Rao, and B. Brunton, "AJILE movement prediction: Multimodal deep learning for natural human neural recordings and video," in Proceedings of the 32nd AAAI Conference on Artificial Intelligence, 2018.

[31] M. Völker, L. D. Fiederer, S. Berberich, J. Hammer, J. Behncke, P. Kršek et al., "The dynamics of error processing in the human brain as reflected by high-gamma activity in noninvasive and intracranial EEG,' Neurolmage, 2018

[32] T. Pistohl, A. Schulze-Bonhage, A. Aertsen, C. Mehring, and T. Ball, "Decoding natural grasp types from human ecog," Neuroimage, vol. 59, no. 1, pp. 248-260, 2012

[33] A. C. Evans, S. Marrett, P. Neelin, L. Collins, K. Worsley, W. Dai, S. Milot, E. Meyer, and D. Bub, "Anatomical mapping of functional activation in stereotactic coordinate space," Neuroimage, vol. 1, no. 1, pp. 43-53, 1992.

[34] S. B. Eickhoff, K. E. Stephan, H. Mohlberg, C. Grefkes, G. R. Fink, $\mathrm{K}$. Amunts, and K. Zilles, "A new spm toolbox for combining probabilistic cytoarchitectonic maps and functional imaging data," Neuroimage, vol. 25, no. 4, pp. 1325-1335, 2005.

[35] O. Ledoit and M. Wolf, "A well-conditioned estimator for largedimensional covariance matrices," Journal of multivariate analysis, vol. 88 , no. 2 , pp. 365-411, 2004.

[36] V. J. Lawhern, A. J. Solon, N. R. Waytowich, S. M. Gordon, C. P. Hung, and B. J. Lance, "EEGNet: A Compact Convolutional Network for EEGbased Brain-Computer Interfaces," arXiv preprint arXiv:1611.08024v2, Nov. 2016.

[37] I. Loshchilov and F. Hutter, "Fixing weight decay regularization in adam," arXiv preprint arXiv:1711.05101, 2017.

[38] — "SGDR: Stochastic gradient descent with warm restarts," arXiv preprint arXiv:1608.03983, 2016.

[39] F. Wilcoxon, "Individual comparisons by ranking methods," Biometrics bulletin, vol. 1 , no. 6, pp. 80-83, 1945.

[40] R. Chavarriaga, A. Sobolewski, and J. d. R. Millán, "Errare machinale est: the use of error-related potentials in brain-machine interfaces," Frontiers in neuroscience, vol. 8, p. 208, 2014.

[41] D. G. Altman and J. M. Bland, "Diagnostic tests. 1: Sensitivity and specificity." BMJ: British Medical Journal, vol. 308, no. 6943, p. 1552 , 1994.

[42] D. D. Lewis, "An evaluation of phrasal and clustered representations on a text categorization task," in Proceedings of the 15th annual international ACM SIGIR conference on Research and development in information retrieval. ACM, 1992, pp. 37-50.

[43] J. Mazziotta, A. Toga, A. Evans, P. Fox, J. Lancaster, K. Zilles, R. Woods, T. Paus, G. Simpson, B. Pike et al., "A probabilistic atlas and reference system for the human brain: International consortium for brain mapping (icbm)," Philosophical Transactions of the Royal Society B: Biological Sciences, vol. 356, no. 1412, pp. 1293-1322, 2001.

[44] F. Tadel, S. Baillet, J. C. Mosher, D. Pantazis, and R. M. Leahy, "Brainstorm: a user-friendly application for meg/eeg analysis," Computational intelligence and neuroscience, vol. 2011, p. 8, 2011.

[45] T. Eichele, S. Debener, V. D. Calhoun, K. Specht, A. K. Engel, K. Hugdahl, D. Y. von Cramon, and M. Ullsperger, "Prediction of human errors by maladaptive changes in event-related brain networks," Proceedings of the National Academy of Sciences, vol. 105, no. 16, pp. 6173-6178, 2008.

[46] J. J. Tecce, "Contingent negative variation (cnv) and psychological processes in man.” Psychological bulletin, vol. 77, no. 2, p. 73, 1972.

[47] M. L. Padilla, R. A. Wood, L. A. Hale, and R. T. Knight, "Lapses in a prefrontal-extrastriate preparatory attention network predict mistakes,' Journal of cognitive neuroscience, vol. 18, no. 9, pp. 1477-1487, 2006.

[48] K. J. Miller, K. E. Weaver, and J. G. Ojemann, "Direct electrophysiological measurement of human default network areas," Proceedings of the National Academy of Sciences, vol. 106, 2009.

[49] K. R. Ridderinkhof, S. Nieuwenhuis, and T. R. Bashore, "Errors are foreshadowed in brain potentials associated with action monitoring in cingulate cortex in humans," Neuroscience letters, vol. 348, no. 1, pp. $1-4,2003$.

[50] J. F. Cavanagh, M. X. Cohen, and J. J. Allen, "Prelude to and resolution of an error: Eeg phase synchrony reveals cognitive control dynamics during action monitoring," Journal of Neuroscience, vol. 29, no. 1, pp. 98-105, 2009.

[51] S. Shipp, R. A. Adams, and K. J. Friston, "Reflections on agranular architecture: predictive coding in the motor cortex," Trends in neurosciences, vol. 36, no. 12, pp. 706-716, 2013.

[52] P. Ramkumar, B. Dekleva, S. Cooler, L. Miller, and K. Kording, "Premotor and motor cortices encode reward," PloS one, vol. 11, no. 8 , p. e0160851, 2016.

[53] N. S. Narayanan and M. Laubach, "Top-down control of motor cortex ensembles by dorsomedial prefrontal cortex," Neuron, vol. 52, no. 5, pp. 921-931, 2006. 